\title{
Vulnerability Assessment for Interdependent Gas and Electricity Networks
}

\author{
Christina Jaworsky \\ Department of Mechanical \\ Engineering \\ Massachusetts Institute of \\ Technology \\ Cambridge MA 02139 \\ jaworsky@mit.edu
}

\author{
Catalina Spataru \\ Energy Institute \\ University College London \\ London UK \\ c.spataru@ucl.ac.uk
}

\author{
Konstantin Turitsyn \\ Department of Mechanical \\ Engineering \\ Massachusetts Institute of \\ Technology \\ Cambridge MA 02139 \\ turitsyn@mit.edu
}

\begin{abstract}
Strong coupling between the gas and electricity infrastructure introduces new kinds of vulnerabilities in energy systems. Identification and assessment of these vulnerabilities is a challenging task because of the complexity of the system and the non-trivial role of the weather, which is inherently random. Prolonged periods of cold weather can dramatically amplify the negative effect of common failure of the equipment. We propose a methodology for identification of the most dangerous scenarios that combine the outage of individual system components with unfavorable weather conditions in a system of coupled gas and electricity. The feasibility of the approach is illustrated on simulations of a detailed model of coupled Gas and Electricity infrastructures in Europe.
\end{abstract}

\section{Introduction}

The role of natural gas in the global energy system has increased dramatically in the recent years. Both in US and in many European countries, the share of natural gas as a primary electricity fuel has increased almost by an order of magnitude in the last two decades. The advancements of shale gas extraction and liquefaction technologies have also made it one of the most attractive primary energy resources. Natural gas based energy can play a critical role in achieving the ambitious plans on reducing the emissions and raising the share of renewable energy in the overall generation mix. Natural gas turbines are some of the most maneuverable generators and their flexibility allows them to mitigate the intermittency of the renewable wind and solar resources.

Heavy reliance on natural gas as a primary energy resource introduces new kinds of risks in the energy system. Unlike electric power that transfers the energy with the speed of light, the velocity of the gas in pipelines is about $5-10 \mathrm{~m} / \mathrm{s}$, with a maximum of 20 $\mathrm{m} / \mathrm{s}$ allowed for intermittent operation. For a several thousand $\mathrm{km}$ pipeline between the natural gas extraction and consumption sites, this corresponds to a several days lag of the global natural gas supply control system. This problem is alleviated in the energy networks by introducing gas storage sites that enable a more rapid response of the system.

The lack of coordination of operation between gas and electricity industries and the lack of gas storage capacity increases the risk of power outages in scenarios of prolonged periods of anomalously high gas consumption. Without coordination, cold weather conditions and/or component outages compromising the alternative electricity supply sources are dangerous for coupled energy systems (see [1] for extensive discussion of the issue). Proper assessment of these risks and operational policies that minimize them are essential for the resilient operation of the energy systems reliant on natural gas.

The contribution of this work is a methodology for the assessment of risk in interconnected but interdependent electricity and gas networks. Our approach uses a probabilistic analysis of extreme weather patterns, a detailed model of the interdependencies between the electricity and gas infrastructures, and an optimization approach for identifying of the most dangerous weather and outage events. Our methodology is computational efficient compared to traditional Monte Carlo approaches to sampling events, allowing us to explore the immensely large space of possible dangerous scenarios in relatively short time. The ability to find the most dangerous scenarios in a small number of operations allows us to use the algorithm for arbitrarily sophisticated models of coupled infrastructures.

After describing our algorithm, we propose specific ways of implementing the probabilistic weather model, modeling the interdependent infrastructures, and implementing the optimization algorithm for finding the high risk scenarios. We validate the approach via simulations of a European energy 
system model and briefly discuss the results of these simulations. We also discuss the potential extensions of the approach and ways of integrating its implementation in policy, planning, and operation decision-making processes.

\section{Challenges in risk assessment of interdependent infrastructures}

Security assessment for power system operations has been studied for many decades and resulted in well-defined standards enforced by the regulating agencies, such as North American Reliability Corporation, on the independent system operators [2]. System operators are required to operate under the conditions where the failure of any individual component does not lead to loss of stability or violations of technological constraints. Moreover, the operators need to maintain a contingency response plan and be prepared for any N-2 events that can destabilize the system or lead to violation of voltage current safety constraints. To our knowledge, the operators are only required to consider the contingencies associated with the loss of power equipment and operate under the conditions of firm supply of fuel for the generators.

The impact of natural gas infrastructure related contingencies on power system operation has been addressed in a number of recent studies. For example, in [3] and [4] a model of interdependent infrastructures that incorporates the gas network constraints in the framework of security constrained unit commitment is introduced. Follow-up works have explored the impact of gas infrastructure on the overall system efficiency and proposed a number of market based coordination strategies. Other relevant studies have addressed the influence of natural gas infrastructure on planning future electric power generation [4-7], the impact of natural gas networks on power system reliability [8,9], and the optimal operation of integrated energy systems through power studies $[10,11]$.

Although security constrained operations can dramatically reduce the frequency of service interruption, they cannot completely prevent the failure scenarios. Further mitigation of risks is possible only when the specific scenarios and most vulnerable elements of the grid are identified and operation planning accounts for those vulnerabilities. The process of the contingency identification is extremely challenging because of the enormous number of factors that affect the energy system operation and the large number of individual failure scenarios that can lead to violation of key constraints. Weather can directly impact the operations, but it is usually not incorporated in the security assessments. Like component failure, weather events can trigger blackouts. For example, prolonged cold can lead to the exhaustion of local gas storage facilities, causing a fuel shortage for electric generators. Without enough generation, the system could destabilize and blackouts could occur.

In normal operation, the electricity and gas utilities adjust their operations to account for weather patterns and maintain reliable service. However, a combination of extreme weather and an outage of a critical system element can cause a situation where a feasible solution meeting all system requirements for optimal system operation doesn't exist. Analysis contingencies that arise from a combination of gas and electricity infrastructure failure and unfavorable weather events is not currently incorporated either in planning or in operation decision-making processes. Management of these risks requires both the adjustment in existing policies and regulations as well as the development of assessment tools capable of rapidly identifying these contingencies.

Unlike contingencies created by the failure of grid components, the inherently dynamic nature weather patterns and response of the energy system to bad weather makes the direct enumeration of contingencies practically infeasible and invalidates the standard approaches to system reliability based on fault trees analysis. We propose a more appropriate approach based on Monte-Carlo sampling from the statistical weather pattern models and random component outages coupled with simulations of energy system response to the events. However, a Monte Carlo approach is also difficult to use in practice, due to high complexity of power system models and the relatively low probability of major contingencies. Therefore, we use a type of importance sampling to find the contingencies that are most probable and most likely to cause blackouts in the grid. By sampling for the most important events, we can reduce the computational time of running simulations. This is especially important because full simulations of a week of time evolution of the interconnected system could take 10 minutes to run. By selectively choosing scenarios of interest, we avoid wasting computational time simulating scenarios where no blackouts occur.

The approach proposed in this paper provides a computationally efficient way of finding the most dangerous scenarios. The method we propose can provide lower bounds on the probability of system failure. Our approach relies on the existence of prior probabilistic distributions for weather patterns in the system and an energy system model capable of simulation of the system response to given weather 
conditions. In the following sections we describe in more details our approach to modeling of weather pattern probabilities, simulation of interconnected gas-electricity infrastructures and, finally provide more details on the sampling method described above.

\section{Methodology 3.1 General System Model}

The instant state of the system in our model is described by the state vector, $(t)$, which represents the flows of power, gas, and amounts gas stored in different locations in discrete time. The system behavior depends on the weather pattern, described by the vector $\boldsymbol{w}(t)$, which incorporates the nodal information about the local temperatures, wind velocities, solar irradiance and other factors relevant for the local energy consumption and generation. The sequence of outage events that may lead to collapse may be described by the vector $\boldsymbol{o}(t)$, representing the locations and timings of failures. We use the underscore notation to refer to the whole histories of state, weather, and outage vectors, i.e. $\underline{x}(t)=$ $\left[\boldsymbol{x}(0), \boldsymbol{x}(1) \ldots \boldsymbol{x}\left(t_{\max }\right)\right]$

The model of the system establishes a functional relation between the exogenous factors like weather pattern $\boldsymbol{w}(t)$, the system outage events $\boldsymbol{o}(t)$, and the response of the system, characterized by the vector function $\boldsymbol{x}(t)$,

$$
\underline{\boldsymbol{x}}=F(\underline{\boldsymbol{w}}, \underline{\boldsymbol{o}}) .
$$

The function $\mathrm{F}$ incorporates the physical and operational models of the system, including unit commitment, economic dispatch and planning optimal power flow, and transforms the whole history of weather $\underline{\boldsymbol{w}}$ and history of outages $\underline{\boldsymbol{o}}$ into the history of state evolution $\underline{\boldsymbol{x}}$. We describe $\mathrm{F}$ in more detail in Section 3.4.

As long as the weather patterns and outages are modeled as some stochastic processes, the system state $\underline{\boldsymbol{x}}$ is also a stochastic process. The risk assessment problem can be posed as evaluation of expectation of the cost function $I(\underline{x})$ with respect to the probability measures defined of the weather and outage histories. The cost function represents the economic or other losses incurred because by the system during its evolution. The overall risk can be evaluated as the following integral over stochastic process realizations:

$$
\mathrm{E}\{I(\underline{\boldsymbol{x}})\}=\int d \underline{\boldsymbol{w}} d \underline{\boldsymbol{o}} P_{w}(\underline{\boldsymbol{w}}) P_{o}(\underline{\boldsymbol{o}}) I(F(\underline{\boldsymbol{o}}, \underline{\boldsymbol{w}}))
$$

Here the functions $P_{w}(\underline{\boldsymbol{w}}), P_{o}(\underline{\boldsymbol{o}})$ represent the probability density functions of the weather and outage histories. For discrete time, we use a summation over all possible scenarios instead of the integral formulation.

We focus on the conditional expectation defined individually for each outage scenario:

$$
\mathrm{E}\{I(\underline{\boldsymbol{x}}) \mid \underline{\boldsymbol{o}}\}=\int d \underline{\boldsymbol{w}} P_{w}(\underline{\boldsymbol{w}}) I(F(\underline{\boldsymbol{o}}, \underline{\boldsymbol{w}}))
$$

This conditional expectation represents the risks of system associated with specific outages. The same outage of a single power line or generator can have drastically different effect on the system depending on the state of gas storage facilities because the future electricity and gas consumption levels both depend on the weather before and after the outage.

Evaluation of this conditional probability is a much simpler task because it does not involve a search or summation over outage types. However, it can be computationally prohibitive to evaluate $\mathrm{F}$ over all weather scenarios in real life situations when the function $F$ represents a sophisticated evolution of energy system involving complex decision-making and physical processes. Therefore, instead of looking for the average risk, we define a problem of finding the most probable weather pattern which results in high impact $I$ on the system. Formally, we define the following optimization problem:

$$
\begin{aligned}
& \Pi=\max _{w} P_{w}(\underline{\boldsymbol{w}}) \\
& \text { subject to } \mathrm{I}(\mathrm{F}(\underline{\boldsymbol{w}}, \underline{\boldsymbol{o}}))>\mathrm{I}_{\text {crit }}
\end{aligned}
$$

The function $\Pi$ assigns a quantitative indicator for specific outage scenario $\underline{\boldsymbol{o}}$ and impact severity $\mathrm{I}_{\text {crit }}$, which represents the probability of the most probable weather pattern that can cause such a severe impact. The transformation from total risk to unction $\Pi$ reduces the problem of averaging over all possible weather patterns to an optimization problem over weather patterns of finding the most probable pattern that results in an outage of a critical size. For simple $N-1$ type outages representing the failure of a single component of the system, the function $\Pi$ can be thought of as a risk indicator that shows how vulnerable is the system is to failure of a given component. There is no direct relation between $\Pi$ and the actual risk defined by $\mathrm{E}\{I(\underline{\boldsymbol{x}}) \mid \underline{\boldsymbol{o}}\}$, but the value of $\Pi$ can be used as a substitute for the actual risk in the system. For certain probability distributions the Markov inequalities allow one to link the value of $\Pi$ to a lower bound on the actual risk expectation. The exact conditions for existence of such relations will be discussed in forthcoming works on the subject.

\subsection{Probabilistic model of the weather}


One of the most critical steps in the optimization problem described above is the construction of the probability measure function describing the stochastic realization of the weather patterns. Our weather model describes the weather for each zone of the model as a spatially averaged temperature. We construct our model from historical measurements, accessed through the public domain with the WeatherData database interface in Wolfram Mathematica. The database provides the time-series of daily averaged temperature for all major cities. We use the daily averages from major European cities from the years $2000-2013$ to construct our model.

The physical nature of atmospheric processes responsible for weather patterns introduces seasonal trends and strong spatio-temporal correlations of weather in nearby geographic areas. The stochastic model mimicking the actual weather has to incorporate both of these effects. The seasonal trends are reasonably slow in comparison to the timescales relevant for the evolution of interdependent gaselectricity infrastructures. The easiest way to remove the seasonal trends is to construct a separate model for each month of the year. More sophisticated and mathematically rigorous approaches could be based on Seasonal ARIMA or other types of similar statistical models, but have not been used in this work because we only simulate two weeks of system behavior and therefore do not expect seasonal trends to be a strong contributing factor to the results.

It is challenging to incorporate the spatio-temporal correlations of the data in the stochastic weather model. We propose to use the multivariate ARMA models to represent the random variations of weather that occur on day-to-week time scales.

We first find the average temperature in every zone of interest and denote it as the vector $\boldsymbol{\mu}$. The random fluctuations superimposed on this average temperature profile can be decomposed into fixed spatial modes each represented by the vector $\boldsymbol{\phi}_{\boldsymbol{k}}$. The full temperature profile can be then represented with the Karhunen-Loeve expansion as

$$
\boldsymbol{T}(t)=\boldsymbol{\mu}+\sum_{k} \sqrt{\sigma_{k}} \omega_{k}(t) \boldsymbol{\phi}_{k}
$$

Here, the $\omega_{k}(t)$ represents the random amplitude of a given mode, and $\sqrt{\sigma_{k}}$ represents its contribution to the overall temperature profile. We choose the vectors $\boldsymbol{\phi}_{k}$ and the factors $\sigma_{k}$ to be the singular vectors and singular values of the spacial covariance matrix of the temperature estimated from the historical data. This approach allows us to use only few modes to represent accurately the temperature fluctuations across the whole region. In particular for the Europe model, using only the 10 highest singular vectors of the covariance matrix allows us to approximate the temperature full temperature fluctuations with the accuracy of about $90 \%$.

The temporal correlations are incorporated via the stochastic model of the mode amplitudes $\omega_{k}(t)$. We use the standard ARMA model to generate the random amplitudes $\omega_{k}(t)$ and estimate the parameters of each process individually for each of the modes $k$. Formally, this corresponds to the following stochastic model for the amplitude $\omega_{k}(t)$ :

$$
\begin{aligned}
\omega_{k}(t)-\sum_{i=1}^{p} a_{i} \omega_{k}(t-i) & \\
& =e_{k}(t)+\sum_{i=1}^{q} b_{i} e_{k}(t-i)
\end{aligned}
$$

Where the numbers $e_{k}(t)$ are independent normally distributed generators of the process. The coefficients $a_{i}, b_{i}$ parameterize the banded covariance matrix, that can be used to generate random samples of temperature profiles.

The process parameters have been estimated using the FindProcessParameters available in Wolfram Mathematica Statistics toolbox. This process allows us to generate the 1 day resolution time series of the weather profile, creating the spatio-temporal correlations observed in historical data with reasonable accuracy. To increase the temporal resolution of the model and interface the weather generation model with the energy system simulation software we downscale the data using a simple linear interpolation of the hourly profiles of temperature variations. These interpolation schemes could be fitted to represent the historical daily profiles of temperatures available through other data sources.

The proposed stochastic model of temperature variations represents the temperature as a Gaussian random vector. The probability of a given vector can be found using the standard Gaussian function:

$$
-\log P_{T}(\underline{\boldsymbol{T}})=\mathrm{c}-(\underline{\boldsymbol{T}}-\boldsymbol{\mu})^{\top} K^{-1}(\underline{\boldsymbol{T}}-\boldsymbol{\mu})
$$

where $K$ is the covariance matrix of the random process, that can be evaluated via linear transformations of the ARMA process covariance matrices. The constant $c$ corresponds to the normalization of the probability density function and does not affect any calculations.

In practice, the optimization should be performed over the lower dimensional time series of amplitudes $\omega_{k}(t)$ that can be further transformed into the temperature variations using the formulas presented above. It is more convenient to use the logarithm of the probability as an optimization objective. For Gaussian models, this logarithm is a simple quadratic function with explicitly defined Jacobians and Hessians that could be naturally used in first and second order optimization algorithms. 


\subsection{Impact function}

The risk assessment procedure defined in this work is based on the optimization to find the most likely scenario to result in a threshold level of impact. The impact function represents the economic costs incurred by the society due to the critical situation in energy system. Constructing the realistic economic impact of the contingency is an extremely challenging problem, due to high levels of model uncertainty and high computational cost of realistic blackout propagation modeling. Instead, we propose to use computationally simple substitutes for the economic cost that represent the dangerousness of the situation and the expected outcome of the cascade dynamics given the initially overloaded state. These functions can be validated in the future on existing data on blackouts or detailed models of emergency dynamics of energy systems in ways similar to the validation of other phenomenological cascade impact models [12].

This work focuses on the impact of gas shortage on the quality of service provided by the energy system, so we construct the impact function representing the amount of storage available in the system. Assuming that the amounts of stored gas in every zone are denoted by the vector $\boldsymbol{g}$ and the instant rate of change of the stored gas by $\Delta \boldsymbol{g}(t)=\boldsymbol{g}(t)-\boldsymbol{g}(t-1)$, we can introduce the exhaustion rates as the vector $\lambda$ with each component defined as $\lambda_{a}=\max \left(\frac{\Delta g_{a}}{g_{a}}, 0\right)$ with $a$ being the index of the zone. The inverses of the exhaustion rate $\tau_{a}=\lambda_{a}^{-1}$ is an estimate of the time it would take to exhaust the local storage of gas if no changes are made in operation. The natural impact function in this case is simply a norm of the vector $\lambda$ averaging the exhaustion rates over many sites:

$$
I=\|\lambda\|_{p}
$$

For example, for $p=\infty$ this norm corresponds simply to the maximal value of the exhaustion rates over many sites. Other natural extensions of this impact function include the temporally filtered expression for the exhaustion trend estimate and a weighted norm that accounts for the importance of different gas storage sites and their overall effect on the system. After the impact function is constructed, we optimize over possible weather patterns and component failures for the scenarios of highest probability that exceeds the chosen level of impact. In the optimization, we consider the weather before and after the component outages because both affect the response of the system to the component outage.

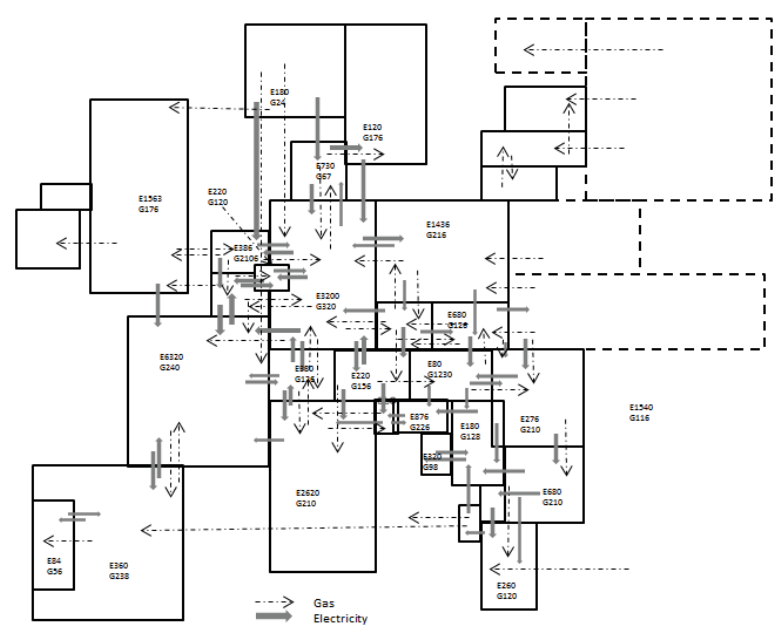

Figure 1 Illustration of zones and inter-connected nodes together with the flows [24]

\subsection{Energy system model}

The energy system model we simulate uses standard DC electricity flow models and flow models for gas. The connections between European countries were modelled as described in [24]. 42 zones, shown in Figure 1, are characterized by the energy gas and power demand profiles. Demands comprise a mix of national electricity consumption and the requirements for imports and exports for storage systems. The instant demand levels for gas and electricity depend on the ambient temperature, taken to be uniform across the whole zone. The zones are linked to each other by a one pipeline which summing up the capacities of all pipelines connecting the zones.

The general approach towards modeling the coupled gas electricity and other forms of networks was described in $[21,22,24]$. In this work, the power flows are modeled with DC approximation with one of the buses chosen as a slack bus. The DC flow equations make sure that the sum of electric generation and imports minus exports at each node equal the demand minus any shedding at that node. Busses between nodes are modeled as lossless in this DC approximation. The demand for power and gas on every node is calculated using the temperature dependent consumption model that was validated on the historical data. The dispatch of generation in each zone is modeled via a heuristic algorithm that accounts for the local demand and supply curves as well as availability of gas storage. The generators are dispatched via a simplified version of optimal power flow that ensures that the power line capacity limit constraints and physical constraints on the ramping of the generators are satisfied while minimizing the 
overall cost of the energy. Once the values of generation and consumption are defined, the power flows are calculated via the admittance matrix defined for the effective power grid.

The electric and gas networks are coupled to each other via gas fired generator units that are modeled as energy converters and are described by a simple relation

$$
P_{g}=E_{f f} Q H_{g}
$$

where $E_{f f}$ is the thermal efficiency of the gas turbines, $H_{g}$ is the gas heating value and $Q$ is the local consumption of gas by the generator unit. The gas pipeline network is modeled as a graph of branches connected to individual zones. The mass flow of gas through each individual pipeline is modeled via a traditional constitutive relation of the form

$$
f_{k}=K_{k}[\Delta \mathrm{Pk}]^{1 / \lambda}
$$

where $f_{k}$ is the flow through the branch, $\Delta \mathrm{Pk}$ is the pressure drop between the nodes connected by the branch and $K_{k}, \lambda$ are some numerical coefficients different for different types of pipelines. The pressure plays a role of effective potential in gas flows and the compressor stations along the pipeline act like transformers that increase the pressure in the pipeline by some fixed factor.

The gas pipeline network is described by a branch nodal incidence matrix $A$, so that the mass conservation law can be written as

$$
\boldsymbol{L}=\boldsymbol{A} \boldsymbol{f}
$$

with $\boldsymbol{L}$ being the vector of nodal loads and $\boldsymbol{f}$ the vector of pipeline flows. An additional unit nodal matrix $U$ describes the interconnection of pipelines to the storage or other units. The third matrix $T$ models the interconnection of generators to the specific nodes. Using these definitions, the overall mass balance equation can be rewritten in the following form:

$$
(A+U) \boldsymbol{f}+\boldsymbol{n}-T \boldsymbol{g}=0,
$$

where we have introduced an additional gas injection vector $\boldsymbol{n}$ for every node and the vector of gas generator unit fuel consumption $\boldsymbol{g}$. The flows through each pipeline are constrained to be lower than the local pipeline capacity limit. This limit is ensured by solving an optimal allocation of gas storage flows that attempts to minimize the exhaustion of the gas while maintaining the branch flows within their limits.

Gas production, gas consumption, storage capacity, gas withdrawal from storage, and cross-border flows are modeled in aggregated way [24]. We assume there is only one facility per country with aggregated capacity and flow rate.
When solving for the time evolution of the state $\underline{x}(\boldsymbol{t})$ of each node in the European model, a cost optimization is performed to minimize the operational costs of responding to demand and weather. Each country is characterized by local time varying demand profile. Energy demand is calculated from annual demand and adjusted to the local temperature profile. The supply is calculated by an iterative solution of the optimal power/gas flow problems, where the optimization procedure attempts to adjust the node import/export rates within the acceptable constraints until the lowest supply cost solution is found. The cost of electricity based on demand and weather is calculated from a historically validated supply cost curve and data on generation capacity, marginal costs and emissions. The cost of energy supply is found at the intersection of the supply curve with the net node demand curve, defined as the difference between overall consumption level and the aggregate import of the energy (negative if the node exports the energy). The optimization procedure incorporates minimizing the rates of storage exhaustion as part of the cost, and generally results in solutions that maximize the utilization of pipelines. Small amounts of randomization are introduced to model by the market and decision-making imperfections.

The simulation of the gas electricity system relies on hybrid optimization module described in [15]. It is based on a combination of genetic algorithm, particle swarm and downhill with three major operators: enhancement, crossover and mutation. In combination these methods are more likely to find the global optimum. By running the optimizer with different starting points, the probability of finding the global optimum is increased. The key advantage of this approach is its ability to avoid single local minima, as initialization with multiple starting points allows the system to explore the non-trivial extrema of the cost function.

\section{Scenarios and results}

\subsection{Base scenario and model validation}

We consider scenarios spanning 14 days with the outage of the component happening right between days 7 and 8 . The model is validated on the base 14 days winter period scenario without any outages happening during the period. We use 14 days in February of 2012 as our base scenario. 


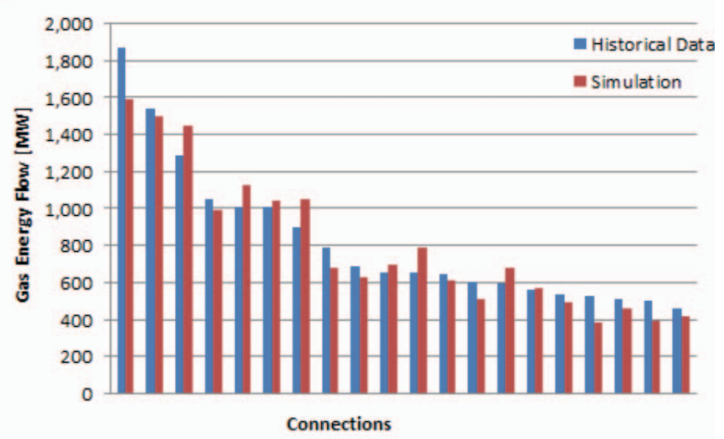

Figure 2 Gas flows for the 20 highest volume pipelines

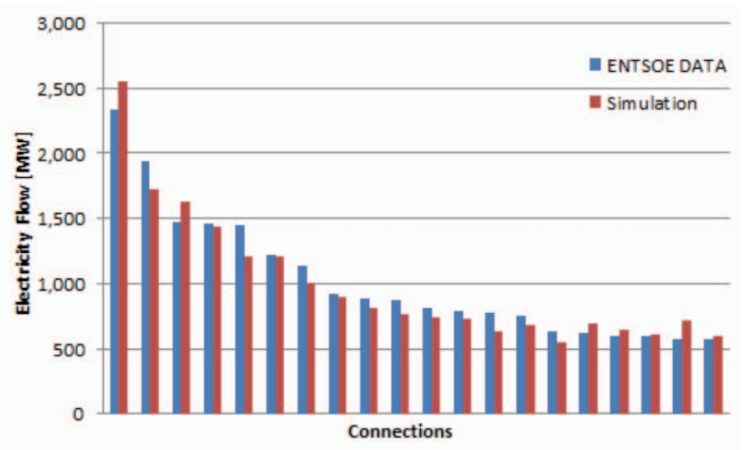

Figure 3 Electricity flows for the 20 highest volume lines

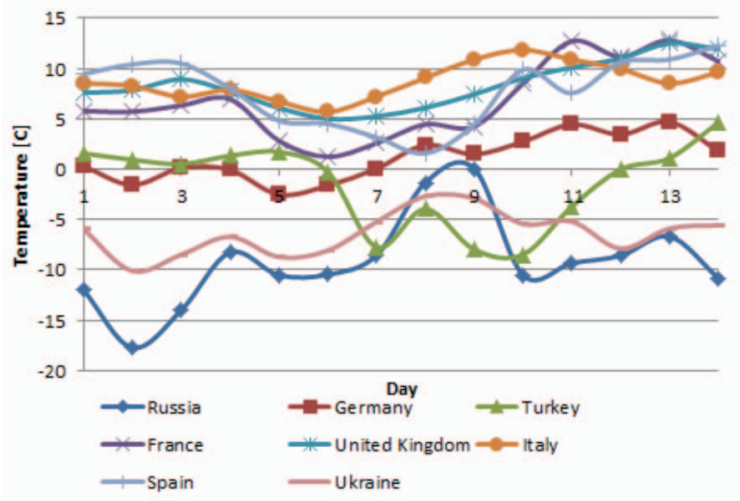

Figure 4 Temperature profile for the most populous of the 42 European zones for the 14 day base scenario.

The average gas consumption was $27,644 \mathrm{GWh} / \mathrm{d}$ during the 14-day period, an increase of $12 \%$ over the same period over the previous winter. The European peak-day demand $(29,141 \mathrm{GWh})$ for the winter 201112 was recorded on the $7^{\text {th }}$ of February 2012. At the country level the differences vary from $-26 \%$ (Ireland) to $+28 \%$ (Germany) [16]. The demand peak period was 31/01/2012-13/02/2012. Historical publicly available data for gas and electricity flows are taken from ENTSOG and ENTSOE.
After simulating our zoned DC flow model with the historical demand and temperature profiles, we find good agreement between the model prediction and the actual cross border flows of gas and electricity. Figures 2 and 3 present the differences from the model prediction and the actual flow data, showing an agreement with only $9.5 \%$ error. The temperature profiles for the base scenario are shown on Figure 4.

\subsection{Disruption scenario: loss of a gas pipeline in Ukraine}

To illustrate the effectiveness of our model and impact function, we consider one scenario. In this scenario, we optimize for high impact weather conditions by considering a cold two week period in winter for all zones in the model. This cold period is accompanied by the failure of one of the key pipelines that is used to supply fuel and heating gas to most of the zones. The motivation behind the scenario was driven by the previous disputed situations between Russia and Ukraine. In 2009 January the $1^{\text {st }}$, deliveries of $90 \mathrm{mmcm}$ of natural gas per day were fully cut and a volume of $300 \mathrm{mmcm}$ per day cut for transit deliveries to EU [26].

Currently, there are three main suppliers of pipeline gas to the EU - Russia, Norway, and Algeria - along with several other suppliers of liquefied gas, most notably Qatar. Russia is the largest single supplier to the EU in 2012, providing $36.5 \%$ of the EU gas imports. Both the EU and Russia are dependent on fixed pipelines for the transit of gas.

There are four main routes for Russian gas exports to the EU - direct pipelines to Finland and the Baltic States, the Nord Stream pipeline under the Baltic Sea to Germany and transit pipelines via Belarus and Ukraine.

In the proposed scenario, we assume at the same time that there is high demand due to low temperature that is accompanied by an outage of the major gas pipeline due to loss of structural integrity (mechanical damage, corrosion, overpressure, etc.) on the Ukraine route which shutdown the Ukrainian transit, producing serious shortages in numerous countries in Europe. For this scenario we generate the samples of low temperature profile using the stochastic model described in the previous sections and run the simulations for different scenarios until the scenario with a high level of impact is found. 


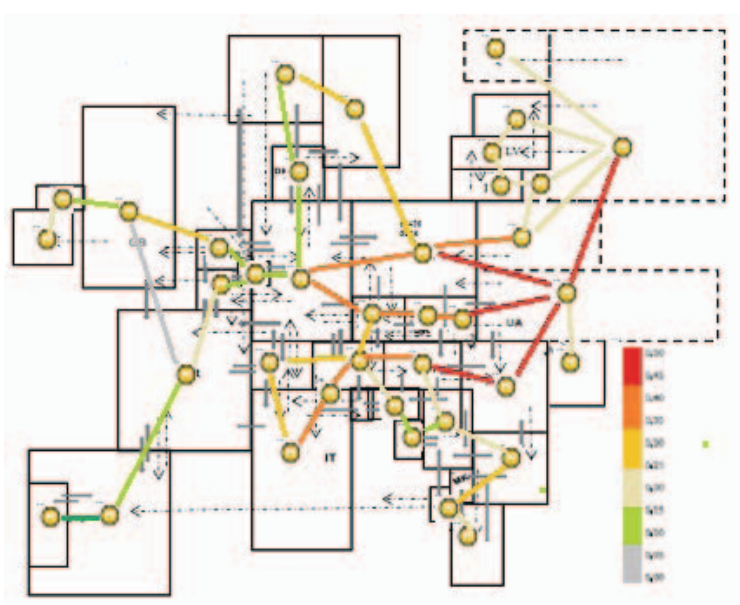

Figure 5 Visualization of the system in the disruption scenario.

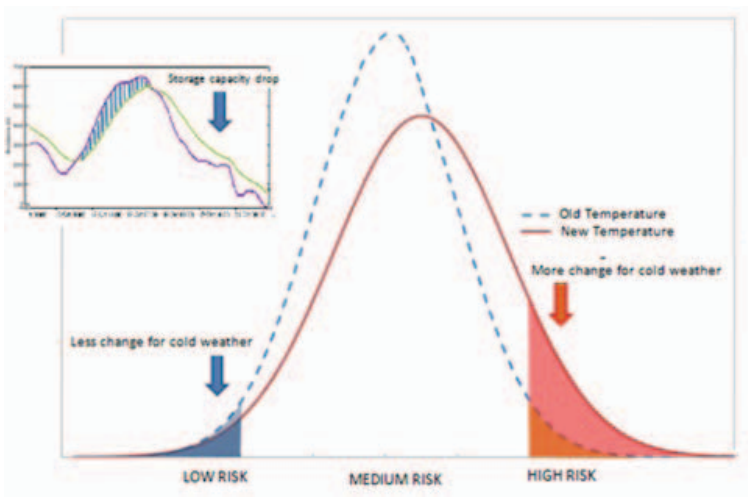

Figure 6 Depletion of the storage in disruptive scenario and effect of the temperature on the risk distribution in the system

Our simulations have shown that the amount of gas stored in the system was rapidly decreasing for most of the sites in the system. To quantify the effect of gas shortage we have used the risk indicator similar to the exhaustion rate defined in previous sections, but adopted to the details of the model of the energy system. The risk indicator accounts for the capacities of all gas sources of a given node (storage and pipelines) as well as the consumption rates observed during the simulations and accounts for the inherent randomness incorporated in the model via MonteCarlo averaging over possible flows for a given temperature profile. We observe that in comparison to the base scenarios, the number of the high risk zones is quite dramatically increased in comparison to the base scenario. Figure 5 shows the color coded map of the system in the disruption scenario, while Figure 6 qualitatively shows storage decay curves during this scenario.

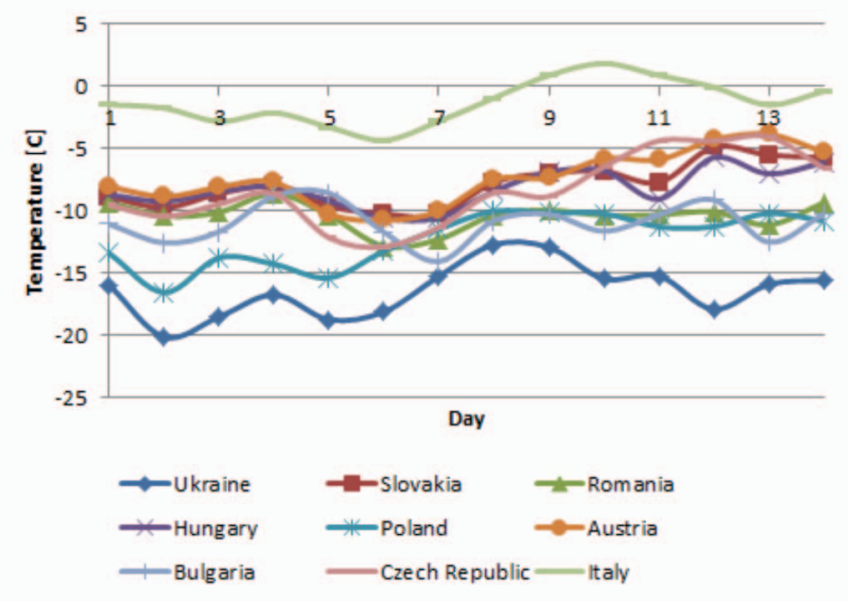

Figure 7 Proposed temperature profile for 14 day scenario with pipeline disruption

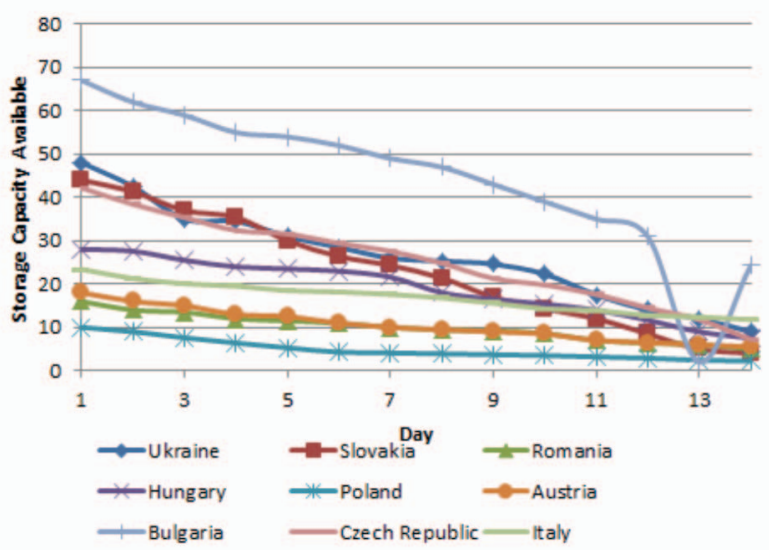

Figure 8 Storage capacity during the disruption and cold weather in the most affected countries

We observe that the countries facing the highest levels of imbalance and storage depletion are:

Hungary, Romania, Slovakia, Poland with the follow rates: $54 \mathrm{Mcm} /$ day, $29 \mathrm{Mcm} /$ day, $26 \mathrm{Mcm} /$ day, 9 $\mathrm{Mcm} /$ day. The effect of low temperature profiles in these countries, shown in the Fig. 7, together with the limited supply from the failed Ukrainian pipeline results in rapid exhaustion of the gas storage, shown in the Fig. 8.

This effect also propagates to other countries: Austria, Italy, Greece, Germany all rely on the gas from Russia. However, the effect on the storage in those countries is less severe due to existence of alternative routes and higher flexibility in supply options. Historically, these countries also were affected by the change in policy between Russia and the Ukraine. When the Russia-Ukraine dispute took place in January 2009, various countries were affected. For example Austria 66\% cut, Bulgaria $100 \%$, Croatia $40 \%$, Czech Rep $71 \%$, Germany $10 \%$, 
Greece $80 \%$, Italy $25 \%$, Hungary $45 \%$, Poland $33 \%$, Slovakia 97\%, Slovenia 50\% [27]. They have to look for alternatives. Austria used the gas in storage for several weeks, Bulgaria the same that they had enough for only $35 \%$ of gas demand which means the equaivalent of 2-3 days, Croatia increased the domestic production by $43 \%$ and storage withdrawal. If we look at the current situation in Europe, according to IEA Gas Trade Flows in Europe, Nord Stream website, in 2012 there was a total of 208.7 $\mathrm{mcm} /$ day of Russian gas flows via Ukraine, this represents about 54\% of exports from Russia to Europe. This indicates clearly that if there is a supply shortfall due to a disruption on the Ukraine route could cause severe disruptions because the system cannot sustain the winter demand under extreme temperature conditions of $-20^{\circ} \mathrm{C}$. In addition, a deficit on the gas supply may have impact on both networks. In countries producing a lot of electricity from gas, such as Italy (with $50 \%$ of its electricity produced from gas powered turbines) the loss of gas supply can be critical.

The situation may turn more severe if there is simultaneous lack of gas and deficit of renewable energy. These two factors combined may result in even higher stress on the system. However this type of propagation of failures is likely to occur only in specific countries with significant electricity generation from gas but with a lack of power connections or lack of gas storage. One natural solution to this problem is reallocation of the delivery paths that originate in Russia through alternative routes through Norway and Netherlands, complemented by an increase of gas storage capacity in the most vulnerable European countries.

\section{Future work}

The approach proposed in this work can be extended in several directions. Existing reliance on Gaussian modeling of the temperature variations is overly restrictive and may be inappropriate for some characteristics like wind velocity or solar irradiance with heavy non-Gaussian probability tails. In the future we plan to extend the technique to work with advanced stochastic weather generators [17] developed by atmospheric research community. These weather generators produce random samples of weather in a given time period, and could be coupled with some stochastic optimization or advanced sampling techniques, like importance sampling. In this framework the system would be composed of three modules: weather generator, energy system simulator and sampling/optimization interfaces that performs optimization on the space of weather samples.

The energy system model should be also improved to incorporate more realistic representation of real life decision-making processes implemented by system operators. For example, gas market and more general contract models are necessary to model the system response in deregulated environments. Similarly the dispatch of generators should account for security constraints imposed by NERC and other regulating entities.

\section{Conclusions}

This paper addressed the problem of resilient operation of interconnected and interdependent gas and electricity infrastructures. Heavy reliance on natural gas introduces additional vulnerabilities in the energy system associated with the shortage of fuel for gas-fired generators. Assessment of the risks associated with these vulnerabilities is especially challenging task, as typical dangerous events involve both the failure of some system components and unfavorable weather conditions. Enormous number of possible failure scenarios makes the straightforward approaches for reliability assessment nearly impractical.

We have proposed a novel approach that is based on the identification of the most probable of the dangerous weather patterns and provided specific ways of how this approach could be implemented in practice. The potential of our ideas was illustrated by adaptation of a complex European energy system model and preliminary analysis of the risks associated with the failure of specific gas pipelines combined with unfavorable weather conditions.

\section{Acknowledgements}

We acknowledge support of MIT/Skoltech initiative for funding this project.

We would also like to thank to British Council for funding support as part of the Internationalising Higher Education' programme.

\section{References}

[1] Report from MIT Energy Initiative symposium: Growing Concerns, Possible Solutions The Interdependency of Natural Gas and Electricity System, April 2013.

[2] NERC Reliability standards, available online at http://www.nerc.com/pa/stand/Pages/ReliabilityStand ardsUnitedStates.aspx?jurisdiction=United $\% 20$ States 
[3] Li, Tao, Mircea Eremia, and Mohammad Shahidehpour. "Interdependency of natural gas network and power system security." Power Systems, IEEE Transactions on 23.4 (2008): 1817-1824.

[4] M. Shahidehpour, Y. Fu, and T. Wiedman. Impact of natural gas infrastructure on electric power systems. Proceedings of the IEEE, 93(5):1042-1056, May 2005.

[5] M.S. Morais and J.W. Marangon Lima. Combined natural gas and electricity network pricing. Electric Power Systems Research, 77(5-6):712 -719, 2007.

[6] L.A. Barroso, B. Flach, R. Kelman, B. Bezerra, S. Binato, J.M. Bressane, and M.V. Pereira. Integrated gas-electricity adequacy planning in Brazil: technical and economical aspects. In Power Engineering Society General Meeting, 2005. IEEE, volume 2, pages 1977-1982, June 2005.

[7] S. Hecq, Y. Bou_oulx, P. Doulliez, and P. Saintes. The integrated planning of the natural gas and electricity systems under market conditions. In Power Tech Proceedings, 2001 IEEE Porto, volume 1, page 5 pp., 2001.

[8] J. Munoz, N. Jimenez-Redondo, J. Perez-Ruiz, and J. Barquin. Natural gas network modeling for power systems reliability studies. In Power Tech Conference Proceedings, 2003 IEEE Bologna, volume 4, page 8 pp., June 2003.

[9] A. Helseth and A.T. Holen. Reliability modeling of gas and electric power dis- tribution systems; similarities and differences. In Probabilistic Methods Applied to Power Systems, 2006. PMAPS 2006. International Conference on, pages 1-5, June 2006.

[10] C. Unsihuay, J.W.M. Lima, and A.C.Z. de Souza. Modeling the integrated natural gas and electricity optimal power. In Power Engineering Society General Meeting, 2007. IEEE, pages 1-7, June 2007.

[11] M. Chaudry, N. Jenkins, and G. Strbac. Multitime period combined gas and electricity network optimisation. Electric Power Systems Research, 78(7):1265 - 1279, 2008.

[12] Carreras, Benjamin A., David E. Newman, Ian Dobson, and Naga S. Degala. "Validating OPA with WECC data." In System Sciences (HICSS), 2013 46th Hawaii International Conference on, pp. 2197-2204. IEEE, 2013.

[13] S. An, Q. Li, T.W. Gedra, "Natural gas and electricity optimal power flow", Transmission and Distribution Conference and Exposition, 2003 IEEE Power Engineering Society, vol. 1, 7-12 pp. 138-143, 2003

[14] M. Arnold, G. Andersson, "Decomposed electricity and natural gas optimal power flow", presented at the 16th Power Systems Computation Conference (PSCC 08), Glasgow, Scotland, 2008

[15] C. Spataru, M. Barrett, The importance of optimization and controls in future intelligent grids, IEEE Powertech Grenoble, pp 1-6, 2013

[16] European Network of Transmission System Operators for Electricity 'August 2009 Monthly Provisional Values as of $8^{\text {th }}$ January 2011' Available at https://www.entsoe.eu/resources

[17] Semenov, Mikhail A., and Elaine M. Barrow. "Use of a stochastic weather generator in the development of climate change scenarios." Climatic change 35, no. 4 (1997): 397-414

[18] Seungwon An, Qing Li, and T.W. Gedra. Natural gas and electricity optimal power. In Transmission and Distribution Conference and Exposition, 2003

IEEE PES, volume 1, pages 138-143, Sept. 2003.

[19] C. Unsihuay, J.W.M. Lima, and A.C.Z. de Souza. Modeling the integrated natural gas and electricity optimal power. In Power Engineering Society General

Meeting, 2007. IEEE, pages 1-7, June 2007.

[20] M. Chaudry, N. Jenkins, and G. Strbac. Multitime period combined gas and electricity network optimisation. Electric Power Systems Research, 78(7): 1265 - 1279, 2008.

[21] S. An, Q. Li, T.W. Gedra, "Natural gas and electricity optimal power flow", Transmission and Distribution Conference and Exposition, 2003 IEEE Power Engineering Society, vol. 1, 7-12 pp. 138-143, 2003

[22] M. Arnold, G. Andersson, "Decomposed electricity and natural gas optimal power flow", presented at the 16th Power Systems Computation Conference (PSCC 08), Glasgow, Scotland, 2008

[23] C. Spataru, M. Barrett, "The importance of optimization and controls in future intelligent grids", IEEE PowerTech (POWERTECH), Grenoble, pp 1-6, 2013, DOI: 10.1109/PTC.2013.6652171

[24] C. Spataru, J. Bialek Energy Networks: A Modelling Framework for European Optimal CrossBorder Trades, 2014 IEEE PES General Meeting [25] European Network of Transmission System Operators for Electricity 'August 2009 Monthly Provisional Values as of $8^{\text {th }}$ January 2011' Available at https://www.entsoe.eu/resources

[26] Russia fully cuts gas to Ukraine, ups supplies to Europe. RIA Novosti. January 1, 2009. Retrieved January 1, 2009.

[27] The Russo-Ukrainian gas dispute of January 2009: a comprehensive assessment page 53-55 Oxford Institute for Energy Studies, Retrieved on October 13, 2009 